\title{
Phytochemical profiling of petroleum ether and chloroform extracts of Curcuma caesia rhizome by GC-MS and comparing their bioactivities
}

\author{
Rameshor S Atom ${ }^{1,2}$, Shaukat Ali M Shaikh ${ }^{3}$, Warjeet S Laitonjam ${ }^{2,}$, Raghumani S Ninghthoujam $^{4}$ \\ \& Amit Kunwar ${ }^{5, *}$ \\ ${ }^{1}$ Department of Chemistry, Waikhom Mani Girls College, Thoubal 795138, India. \\ ${ }^{2}$ Department of Chemistry, Manipur University, Imphal 795003, India. \\ ${ }^{3}$ School of Chemical Sciences, UM-DAE, Centre for Excellence in Basic Sciences, University of Mumbai, \\ Kalina Campus, Sant Cruz (East), Mumbai 400098, India. \\ ${ }^{4}$ Chemistry Division, Bhabha Atomic Research Centre, Mumbai 400085, India. \\ ${ }^{5}$ Radiation $\&$ Photochemistry Division, Bhabha Atomic Research Centre, Mumbai 400085, India. \\ E-mail:kamit@barc.gov.in
}

Received 30 August 2021; Revised 06 December 2021; Accepted 16 December 2021

\begin{abstract}
The objective of this study was to characterise the putative phytochemical constituents from petroleum ether (CC-P) and chloroform (CC-C) extracts of Curcuma. caesia rhizome by GC-MS analysis and to compare their bioactivities. GC-MS analysis facilitated the documentation of a total of 16 and 20 volatile constituents in CC-P and CC-C extracts respectively. Of these the major constituents present in CC-P were cycloisolongifolence, 8, 9-dehydro-9 formyl- (19.64\%), 4-oxo$\beta$-isodamascol (16.05\%), 6,10-dimethyl-3- (1-methylethyl) -6-cyclodecene-1,4-dione (13.80\%), 2H-cyclohepta[b]furan-2-one,6-[1-(acetyloxy)-3-oxobutyl]-3,3a,4,7,8,8a-hexahydro-7-methyl3-methylene- (9.08\%), tricycle [5.1.0.0(2,4)]oct-5-ene-5-propanoic acid,3,3,8.8-tetramethyl$(6.39 \%), 2(3 \mathrm{H})$-benzofuranone,6-ethenylhexahydro-3,6-dimethyl-7-(1-methylethenyl)-, [3S- $(3 \alpha, 3 \mathrm{a} \alpha, 6 \alpha, 7 \beta, 7 \mathrm{a} \beta)(4.18 \%)$ and 7a-isopropenyl-4,5-dimethyloctahydroindene-4-carboxylic acid $(4.01 \%)$. Similarly, the major constituents present in CC-C were cycloisolongifolence,8,9dehydro-9-formyl-(15.69\%), 6,10-dimethyl-3-(1-methylethyl)-6-cyclodecene-1, 4-dione (13.38\%), 2H-cyclohepta[b]furan-2-one,6-[1-(acetyloxy)-3-oxobutyl]-3,3a,4,7,8,8a-hexahydro-7-methyl3-methylene- $(4.55 \%)$, tricycle [5.1.0.0(2,4)]oct-5-ene-5-propanoic acid,3,3,8.8-tetramethyl(7.50\%), 2(3H)-benzofuranone,6-ethenylhexahydro-3,6-dimethyl-7-(1-methylethenyl)-,[3S$(3 \alpha, 3 \mathrm{a} \alpha, 6 \alpha, 7 \beta, 7 \mathrm{a} \beta)(6.15 \%), 7 a-i s o p r o p e n y l-4,5-d$ imethyloctahydroindene-4-carboxylic acid $(6.43 \%)$ and 2-(4a,8-Dimethyl-6-oxo-1,2,3,4-4a,5,6,8a-octahydro-naphthalen-2-yl) propionaldehyde $(6.57 \%)$. The extracts, CC-P and CC-C exhibited almost similar antibacterial and antifungal potentials but differed with respect to antioxidant activity. The $\mathrm{IC}_{50}$ values of CC-P and CC-C extracts for DPPH scavenging were $0.68 \pm 0.02 \mathrm{mg} \mathrm{ml}^{-1}$ and $0.13 \pm 0.01 \mathrm{mg} \mathrm{ml}^{-1}$ respectively. Together, above results provide an important basis for the isolation of bioactive compounds from $C$. caesia rhizome extract and their use as traditional medicine.
\end{abstract}

Keywords: phytoconstituents, GC-MS, bioactivities, antifungal, antibacterial, DPPH scavenging 


\section{Introduction}

Plants of Zingiberaceae family are good source of antioxidants and antimicrobial bioactive substances (I-Nan et al. 2008; Kamazeri et al. 2012; Voravuthikunchai 2007). Curcuma. caesia is known as black turmeric for its unique black-bluish rhizome and is endemic to north east and central India (Bhupendra et al. 2016). The plant is cultivated across southeast Asian countries for its innumerable medicinal usage (Arulmozhi et al. 2006; Devi et al. 2015; Gupta et al. 2018; Jain \& Parihar 2018; Jose \& Thomas 2014; Karmakar et al. 2013; Sonjit et al. 2013). This has prompted researchers to document the phyto constituents of rhizome and rhizome oil of $C$. caesia. Occurrence of various compounds in C. caesia rhizome; rhizome oil and leaf essential oil were reported by different researchers (Singh et al. 2010; Mukunthan et al. 2014; Paw et al. 2019; Paliwal et al. 2011; Banarjee \& Nigam 1984; Borah et al. 2019). Apart from oil, plant extracts are also known to be rich in volatile bioactive constituents. A wide range of extraction methods are reported to prepare plant extracts such as microwaveassisted extraction (MAE), ultrasound-assisted extraction (UAE), supercritical fluid extraction (SFE), maceration and Soxhlet extraction (Azwanida, 2015). Of these, Soxhlet method can extract large amounts of phytochemicals with a much smaller quantity of solvent, thus allows tremendous economy in terms of time, energy and cost. Some of the common solvents used for preparing plant extracts include water, methanol, chloroform, petroleum ether, chloroform, ethyl acetate, benzene and hexane among others (Azwanida, 2015). The constituents of plant extracts may differ depending on the extraction solvent used. For example, plant extracts prepared using polar solvents like water, methanol, ethanol and ethyl acetate will be rich in polar compounds whereas those prepared in nonpolar solvents such as chloroform, petroleum ether, benzene and hexane contain mainly non polar compounds. Overall, non polar compounds are biologically more active as compared to polar ones due to their higher cellular uptake and permeability through cell membranes
(Pancharoen et al.2000). Some of the well-known bioactive non polar compounds isolated from plant extract of Zingiberaceae family include curcumin, turmerone and atlantone among others (Pancharoen et al. 2000). The choice of solvent is also known to dictate the toxicity of plant extracts. Among non polar solvents, chloroform and petroleum are preferred over benzene, hexane and others because of their lower toxicity to the biological systems. Thus, choice of the solvent for preparing plant extract is a necessary parameter to be considered for isolating bioactive compounds. There are limited studies on the identification of the volatile constituents of the extract prepared from C. caesia rhizome. Accordingly, the present study was aimed to identify the putative nonpolar volatile constituents from petroleum ether (CC-P) and chloroform (CC-C) extracts of C. caesia rhizome by GC-MS and to study their bioactivities.

\section{Materials and methods}

\section{Reagents and chemicals}

The organic solvents such as chloroform, petroleum ether, methanol and carbon tetrachloride of UV spectroscopy grade was procured from local suppliers. 1,1-Diphenyl-2picrylhydrazyl (DPPH) and soy lecithin were procured from Sigma-Aldrich (Steinheim, Germany). Buffer reagents like monosodium phosphate, disodium phosphate, potassium persulphate $\left(\mathrm{K}_{2} \mathrm{~S}_{2} \mathrm{O}_{8}\right)$ and diammonium salt of 2,2'-azino-bis (3-ethylbenz-thiazoline-6sulfonic acid) (ABTS) of analytical grade were procured from the local suppliers (Ravis \& Company, Mumbai). Nutrient broth, potato dextrose agar medium, potato dextrose broth and dimethyl sulfoxide (DMSO) were obtained from HIMEDIA, India. Nitrous Oxide $\left(\mathrm{N}_{2} \mathrm{O}\right)$ gas of highest purity was used for pursing of liposomal solution of soy lecithin. Reagent solutions were prepared using water from Milli-Q system (Elix-5, MerckMillipore, Germany). Steady state absorption measurements were carried out in a quartz cuvette $(1 \mathrm{~cm} \times 1 \mathrm{~cm})$ by employing JASCO V-630 spectrophotometer (Japan). Further, ${ }^{60} \mathrm{Co}$ $\gamma$-irradiation facility (dose rate $=9$ Gy $\mathrm{min}^{-1}$ ) 
of Bhabha Atomic Research Centre) was used to perform the lipid peroxidation assay. The absorbed dose for the source was validated by standard Fricke dosimetry solution.

\section{Collection of plant material}

The rhizomes of $C$. caesia were collected from Thoubal District, Manipur, North-East India during December 2017.

\section{Extract preparation}

The rhizomes were cleaned under tap water, sliced, air dried (room temperature for 15 days) and ground into fine powder of 3040 mesh size by an electric grinder (USHA Optima Classic 500 W Mixer Grinder, 1800 RPM). Further, $115 \mathrm{~g}$ of the rhizome powder was sequentially extracted with $300 \mathrm{ml}$ each of petroleum ether and chloroform by a Soxhlet extractor at their respective boiling points till colourless solvents were obtained. The solvents from of the respective extracts were evaporated under reduced pressure using vacuum rotary evaporator to obtain viscous semi solid masses, dried at room temperature and stored in air tight containers at $4^{\circ} \mathrm{C}$ until used. The corresponding extracts so obtained were abbreviated as CC-P for petroleum ether extract and CC-C for chloroform extract (Fig. 1).

\section{Phytochemical grouping}

Qualitative phytochemical screening of the extracts was performed using the standard protocol reported earlier (Donipati \& Sreeramulu 2015; Satyavam \& Warjeet 2012). In brief, for alkaloid testing, extract was incubated with mixture of Mayer's reagent, Wagner's reagent and Dragendorff reagent and monitored for precipitation. For flavonoid testing, extract was incubated with magnesium ribbon and monitored for appearance of red or yellow coloration. For phenol detection, extract was mixed with $10 \%$ aqueous $\mathrm{FeCl}_{3}$ and monitored for appearance of blue or green coloration. For tannin detection, extract was mixed with potassium dichromate and monitored for precipitation. For steroid detection, extract was mixed with chloroform- sulfuric acid mixture and monitored for bluish red to cherry coloration. For terpenoids detection, extract was mixed with chloroformsulfuric acid mixture and monitored for reddish brown precipitate. For saponin detection, extract was mixed with distilled water and examined for frothing persistence. For cardiac glycosides, extract was incubated with glacial acetic acid, ferric chloride and concentrated $\mathrm{H}_{2} \mathrm{SO}_{4}$ and monitored for appearance of green coloration. For protein detection, extract was mixed with Millon's reagent and $\mathrm{H}_{2} \mathrm{SO}_{4}$ and monitored for red colour precipitate. For coumarin testing, extract was mixed with 10\% $\mathrm{NaOH}$ and monitored for yellow colouration.

\section{GC-MS study}

GC-MS study was conducted on a GC instrument (Agilent Technologies 7890 A) coupled to mass spectrometer (AccuTOFGCv,JMS-T100GCV, JEOL, Japan) under the following experimental conditions. The instrument was calibrated every six months using perfluorokerosene (b.p. 430). About $1 \mu \mathrm{l}$ of the extract solution in methanol was injected using helium as a carrier gas with a flow rate of $1 \mathrm{ml} / \mathrm{min}$ and split ratio of 1:10. The analytical column attached to the GC was HP5 column (length - $30 \mathrm{~m}$, internal diameter - $0.25 \mathrm{~mm}$, ethylacetate film thickness - 0.25 $\mu \mathrm{M}$, Agilent Technologies India Pvt. Ltd., Bangalore) and the temperature of injector was adjusted at $250{ }^{\circ} \mathrm{C}$. The GC oven temperature program started at $60^{\circ} \mathrm{C}$ (hold for $1 \mathrm{~min}$ ), ramped to $200{ }^{\circ} \mathrm{C}$ at $8^{\circ} \mathrm{C} \min ^{-1}$ (1 min hold), then to $275^{\circ} \mathrm{C}$ at $6{ }^{\circ} \mathrm{C} \mathrm{min}^{-1}$ up (15 min hold), and finally, to $285^{\circ} \mathrm{C}$ at $5{ }^{\circ} \mathrm{C} \mathrm{min}{ }^{-1}$ with $0 \mathrm{~min}$ hold. The total run time of GC analysis was $49 \mathrm{~min}$. The data was acquired using electron ionisation (EI) positive mode at $70 \mathrm{eV}$ and the mass scan was performed from 40 to $700 \mathrm{Da}$. An individual peak separated by GC entered the MS and got ionized. The compounds were putatively identified by matching mass ion peaks corresponding to individual peak of chromatogram with mass ion peaks of known or reference spectra stored in National Institute of Standard and Technology (NIST), MS library Search 2.0-[Q] software. The 


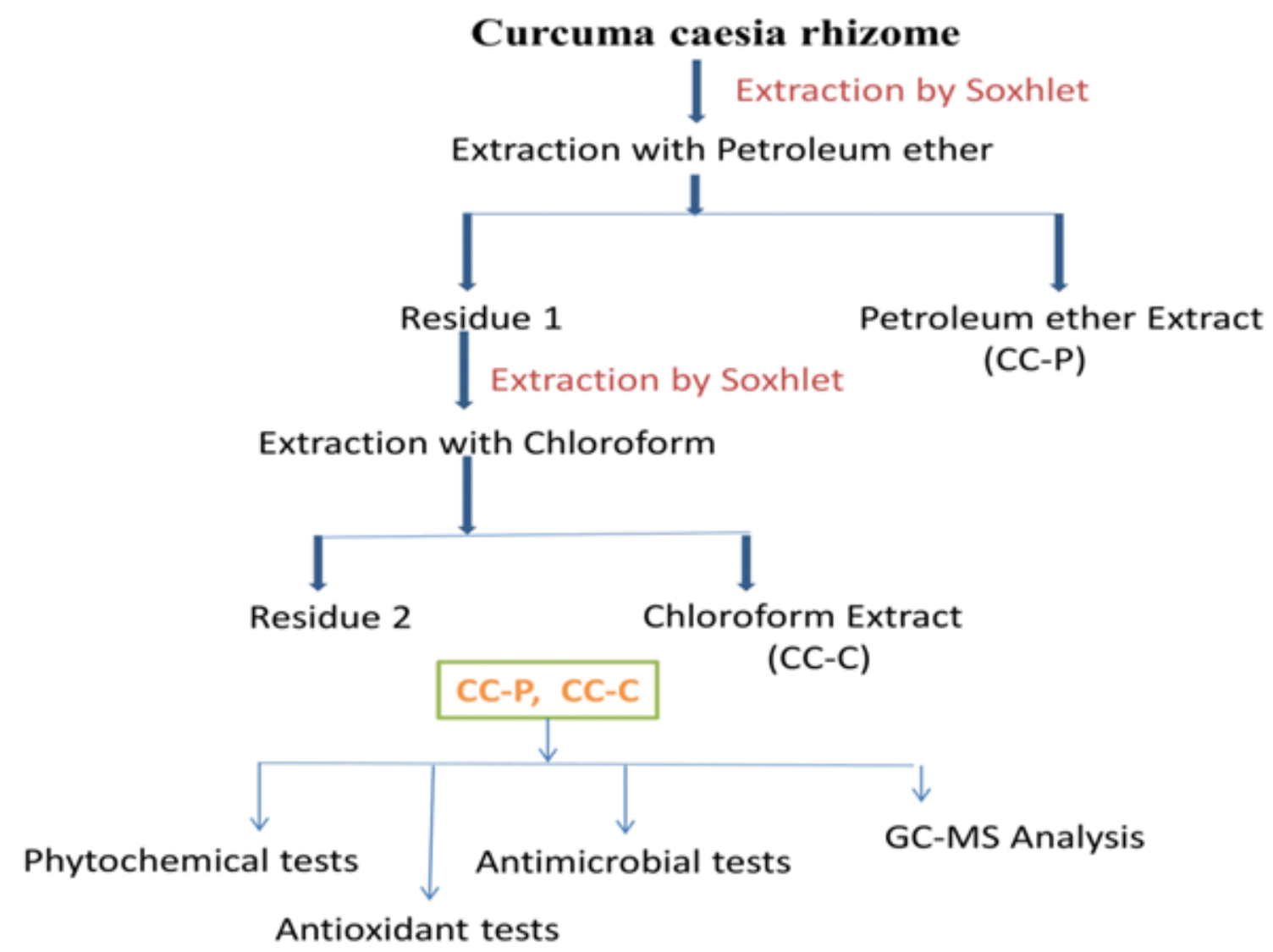

Fig. 1 Schematic diagram depicting stepwise extraction process from the rhizome of C. caesia using petroleum ether and chloroform as organic solvent

library search software of the GCMS system calculated three parameters, match factor (on a scale of 0-999), reverse match factor (on a scale of 0-999), and probability (\% on a scale of $0-100 \%)$ to generate the hit list of putative compounds with possible matching mass spectra, chemical structure and molecular weight. From this list, the compound showing highest match factor score and probability was assigned as the chemical identity of the submitted peak of the chromatogram. The relative abundance of each chromatogram peak was calculated by normalizing its area with the total area of chromatogram.

\section{Antimicrobial and antifungal activities}

\section{Test organisms}

The antibacterial screening was performed against three gram-positive bacteria, Bacillus subtilis (MTCC121), Micrococcus luteus (MTCC 106) and Mycobacterium smegmatis (MTCC 6) and one gram-negative bacteria, Escherichia coli MTCC 739). The antifungal screening was performed against a plant pathogen Rhizoctonia solani (MTCC 4633) and an animal pathogen Aspergillus niger (MTCC 1344). The pathogenic bacterial and fungal strains were procured from IMTECH, Chandigarh, India.

\section{Biocidal assays}

The CC-C and CC-P were evaluated for antifungal and antibacterial activities by agar well diffusion method (Bauer et al. 1966) with some modifications. Crude extracts were solubilized in DMSO to prepare a solution with $25 \mu \mathrm{g} \mathrm{ml}{ }^{-1}$ concentrations. The bacterial test pathogens and fungal test pathogens were grown in nutrient broth and potato dextrose broth respectively for 24 hours at $37^{\circ} \mathrm{C}$ and 
$100 \mu \mathrm{l}$ of the bacterial broths were spread onto nutrient agar media and fungal culture broths onto potato dextrose agar. Sterile cork borer was used to make wells in each plate of $6 \mathrm{~mm}$ diameter. About $100 \mu \mathrm{l}$ of the samples were inoculated in $6 \mathrm{~mm}$ wells and the plates were incubated for 24 hours at $37^{\circ} \mathrm{C}$. Clearing zone around the well indicated antibacterial/ antifungal activity and were compared with the activity of the standards, Ampicillin (25 $\mu \mathrm{g})$ and Fluconazole $(25 \mu \mathrm{g})$. DMSO served as negative control.

\section{Antioxidant activity measurements}

The extracts, CC-P and CC-C were examined for antioxidant activity by measuring the scavenging of ABTS and DPPH radicals and of the inhibition of oxidative degradation of lipids or lipid peroxidation by using methods described previously (Rameshor et al. 2021). Due to low solubility of CC-P and $\mathrm{CC}-\mathrm{C}$ extracts in aqueous system, it was first dissolved in pure methanol and subsequently the percentage of methanol in phosphate buffer ( $\mathrm{pH} \mathrm{7)}$ solutions was adjusted as per the experimental conditions. In brief, DPPH $(50 \mu \mathrm{M})$ or ABTS $(120 \mu \mathrm{M})$ were mixed with the varying concentrations of extracts (CC-P/ $\mathrm{CC}-\mathrm{C})$ in phosphate buffer ( $\mathrm{pH} 7)$, incubated in dark for 20 minutes (room temperature) and subjected to absorbance measurement at $518 \mathrm{~nm}$ and $738 \mathrm{~nm}$ respectively. The $\mathrm{IC}_{50}$ (concentration of extracts which reduces the absorbance of DPPH radicals to half of initial value) were determined from the percentage scavenging activity by using equation (1) as given below.

$\%$ scavenging activity $=\frac{\left(\mathrm{A}_{\text {control }}-\mathrm{A}_{\text {extract }}\right)}{\mathrm{A}_{\text {control }}} \times 100$

Where, $\mathrm{A}_{\text {control }}$ and $\mathrm{A}_{\text {extract }}$ are the absorbance in the absence and in the presence of extracts respectively. For lipid peroxidation, liposomes $\left(1 \mathrm{mg} \mathrm{ml}^{-1}\right)$ from soy lecithin were mixed with $50 \mathrm{\mu g} \mathrm{ml}^{-1}$ of extracts (CC-P/CC-C) in phosphate buffer ( $\mathrm{pH} 7$ ), irradiated using ${ }^{60} \mathrm{Co} \gamma$-radiation (dose rate 9 Gy minute ${ }^{-1}$ ) at an absorbed dose of $270 \mathrm{~Gy}$ and then processed for thiobarbituric acid reactive substances (TBARS) assay (Rameshor et al. 2021). The content of TBARS was determined by monitoring the absorbance at $532 \mathrm{~nm}$ and percent (\%) inhibition was estimated using the following equation:

$$
\% \text { Inhibition }=\frac{\left([\mathrm{TBARS}]_{\text {control }}-[\text { TBARS }]_{\text {extract }}\right)}{[\text { TBARS }]_{\text {control }}}
$$

Where, $[\text { TBARS }]_{\text {control }}$ and $[\text { TBARS }]_{\text {extract }}$ are the concentrations in the absence and presence of extracts (CC-P/CC-C) respectively.

\section{Results \& discussion}

\section{Documentation of phytochemicals}

The plant extracts depending on the extraction method may differ in the medicinal properties. In order to address this, CC-P ( 100 mg kg-1) and CC-C $\left(\sim 125 \mathrm{mg} \mathrm{kg}^{-1}\right)$ extracts were prepared from the rhizome of $C$. ceasia and the preliminary phytochemical examination of these extracts documented the existence of phytochemicals of different classes such as flavonoids, alkaloids, tannins, phenolic compounds, steroids, terpenoids, cardiacglycosides, saponins and coumarin.

Additionally, protein was also found in the CC-P extract. Based on these results, the CC-P and CC-C extracts were further subjected to the GCMS evaluation for putative identification of the volatile constituents of these extracts. The CC-P extract showed a total of 35 peaks, of which 16 matched with NIST library (Table 1). Similarly, the CC-C extract showed a total of 40 peaks, of which 20 matched with NIST library (Table 2). Together, GSMS evaluation revealed the chemical identities of a total of 16 and 20 compounds respectively from the CC-P and CC-C extracts (Tables $1 \& 2$ ). Among the NIST matched compounds, 5,6-Azulenedicarboxaldehyde,1,2,3,3a, 8,8ahexahydro-2,2,8-trimethyl-,(3a $\alpha, 8 \alpha, 8 \mathrm{a} \alpha)-(-)-$; Isoaromadendrene epoxide; 4-Oxo- $\beta$ isodamascol; 4,8,13-Cyclotetradecatriene1,3-diol,1,5,9-trimethyl-12-(1-methylethyl)and 9,12,15-Octadecatrienoic acid-2,3-bis (acetyloxy) propyl ester, $[Z, Z, Z]-$ were 
Table 1. GC-MS identification of phytochemicals present in petroleum ether extract (CC-P) of the rhizome of C. caesia

\begin{tabular}{|c|c|c|c|c|c|c|}
\hline $\begin{array}{l}\text { Sl. } \\
\text { No. }\end{array}$ & RT & $\begin{array}{l}\text { Area } \\
\text { [Intens. sec] }\end{array}$ & $\begin{array}{l}\text { Mass } \\
\text { fragment } \\
\text { fingerprint } \\
(\mathrm{m} / \mathrm{z})\end{array}$ & Name of compound and molecular formula & $\begin{array}{l}\text { Class of } \\
\text { compound }\end{array}$ & $\begin{array}{l}\text { MW } \\
(\mathrm{Da})\end{array}$ \\
\hline 1 & 17.8712 & 5730418.12 & $\begin{array}{l}79,91,108 \\
133,148,216\end{array}$ & $\begin{array}{l}\text { Benzofuran,6-ethenyl-4,5,6,7-tetrahydro-3,6- } \\
\text { dimethyl-5-isopropenyl-,trans- } \\
\left(\mathrm{C}_{15} \mathrm{H}_{20} \mathrm{O}\right)\end{array}$ & Sesquiterpene & 216 \\
\hline 2 & 20.0827 & 55396058.51 & $\begin{array}{l}39,66,91,94 \\
122,123,162 \\
215,230\end{array}$ & $\begin{array}{l}\text { Cycloisolongifolence,8,9-dehydro-9-formyl- } \\
\left(\mathrm{C}_{16} \mathrm{H}_{22} \mathrm{O}\right)\end{array}$ & Sesquiterpene & 230 \\
\hline 3 & 20.4091 & 11318359.69 & $\begin{array}{l}41,55,69,81 \\
93,107,121 \\
136,147,193 \\
236\end{array}$ & $\begin{array}{l}\text { 7a-Isopropenyl-4,5- } \\
\text { dimethyloctahydroindene-4-carboxylic acid } \\
\left(\mathrm{C}_{15} \mathrm{H}_{24} \mathrm{O}_{2}\right)\end{array}$ & Sesquiterpene & 236 \\
\hline 4 & 21.7946 & 4285329.96 & $\begin{array}{l}41,53,67,79 \\
91,107,121 \\
135,136,147 \\
175,203,218\end{array}$ & $\begin{array}{l}\text { 3,7-Cyclodecadien-1-one,3,7-dimethyl-10-(1- } \\
\text { methylethylidene),(E,E)- } \\
\left(\mathrm{C}_{15} \mathrm{H}_{22} \mathrm{O}\right)\end{array}$ & Sesquiterpene & 218 \\
\hline 5 & 22.2343 & 38927338.22 & $\begin{array}{l}41,55,68,82 \\
95,109,121 \\
139,165,167 \\
180,181\end{array}$ & $\begin{array}{l}\text { 6,10-Dimethyl-3-(1-methylethyl)-6- } \\
\text { cyclodecene-1,4-dione } \\
\left(\mathrm{C}_{15} \mathrm{H}_{24} \mathrm{O}_{2}\right)\end{array}$ & Sesquiterpene & 236 \\
\hline 6 & 22.4874 & 18038776.21 & $\begin{array}{l}41,55,67,79 \\
91,105,119 \\
133,147,165 \\
189,196,219 \\
234\end{array}$ & $\begin{array}{l}\text { Tricyclo[5.1.0.0(2,4)]oct-5-ene-5-propanoic } \\
\text { acid,3,3,8.8-tetramethyl- } \\
\left(\mathrm{C}_{15} \mathrm{H}_{22} \mathrm{O}_{2}\right)\end{array}$ & Sesquiterpene & 234 \\
\hline 7 & 22.8471 & 6525373.55 & $\begin{array}{l}41,68,69,82 \\
95,109,121 \\
139,165,167 \\
180,181\end{array}$ & $\begin{array}{l}\text { Neocurdione } \\
\left(\mathrm{C}_{15} \mathrm{H}_{24} \mathrm{O}_{2}\right)\end{array}$ & Sesquiterpene & 236 \\
\hline 8 & 23.4133 & 11801673.62 & $\begin{array}{l}41,67,68,81 \\
96,109,122 \\
164,165,178 \\
234\end{array}$ & $\begin{array}{l}\text { 2(3H)-Benzofuranone,6-ethenylhexahydro- } \\
\text { 3,6-dimethyl-7-(1-methylethenyl)-,[3S- } \\
(3 \alpha, 3 \mathrm{a} \alpha, 6 \alpha, 7 \beta, 7 \mathrm{a} \beta)] \\
\left(\mathrm{C}_{15} \mathrm{H}_{22} \mathrm{O}_{2}\right)\end{array}$ & Sesquiterpene & 234 \\
\hline 9 & 23.5532 & 10695867.24 & $\begin{array}{l}41,53,65,79 \\
91,108,135 \\
162,163,189 \\
203,232,233\end{array}$ & $\begin{array}{l}\text { 5,6-Azulenedicarboxaldehyde, } 1,2,3,3 \mathrm{a}, 8,8 \mathrm{a}- \\
\text { hexahydro-2,2,8-trimethyl-, }(3 \mathrm{a} \alpha, 8 \alpha, 8 \mathrm{a} \alpha)-(-)- \\
\left(\mathrm{C}_{15} \mathrm{H}_{20} \mathrm{O}_{2}\right)\end{array}$ & $\begin{array}{l}\text { Aromatic } \\
\text { compound }\end{array}$ & 232 \\
\hline 10 & 23.7464 & 4306170.71 & $\begin{array}{l}41,53,77,81 \\
93,107,122 \\
123,150,162 \\
215,230\end{array}$ & $\begin{array}{l}\text { Azuleno[4,5-b]furan-2(3H)- } \\
\text { one,3a,4,6a,7,8,9,9a,9b-octahydro-6-methyl- } \\
\text { 3,9-bis(methylene),[3aS-(3a } \alpha, 6 \mathrm{a} \alpha, 9 \mathrm{a} \alpha, 9 \mathrm{~b} \beta)]- \\
\left(\mathrm{C}_{15} \mathrm{H}_{18} \mathrm{O}_{2}\right)\end{array}$ & $\begin{array}{l}\text { Fused } \\
\text { Aromatic } \\
\text { compound }\end{array}$ & 230 \\
\hline 11 & 23.9795 & 1968432.46 & $\begin{array}{l}43,44,67,93 \\
105,121,136, \\
147,159,191 \\
201,232\end{array}$ & $\begin{array}{l}\text { Isoaromadendrene epoxide } \\
\left(\mathrm{C}_{15} \mathrm{H}_{24} \mathrm{O}\right)\end{array}$ & Sesquiterpene & 220 \\
\hline 12 & 24.4658 & 45277656.11 & $\begin{array}{l}41,67,68,69 \\
107,121,167\end{array}$ & $\begin{array}{l}\text { 4-Oxo- } \beta \text {-isodamascol } \\
\left(\mathrm{C}_{13} \mathrm{H}_{20} \mathrm{O}_{2}\right)\end{array}$ & $\begin{array}{l}\text { Substituted } \\
\text { cyclic ketone }\end{array}$ & 208 \\
\hline
\end{tabular}




\begin{tabular}{|c|c|c|c|c|c|c|}
\hline 13 & 26.9238 & 3707821.96 & $\begin{array}{l}43,44,67,79 \\
91,105,123 \\
147,173,201 \\
216,217\end{array}$ & $\begin{array}{l}\text { 6-(1-Hydroxymethylvinyl)-4,8a-dimethyl- } \\
3,5,6,7,8,8 \text { a-hexahydro-1H-naphthalene-2- } \\
\text { one } \\
\left(\mathrm{C}_{15} \mathrm{H}_{22} \mathrm{O}_{2}\right)\end{array}$ & Sesquiterpene & 234 \\
\hline 14 & 27.1835 & 25623614.92 & $\begin{array}{l}43,53,65,77 \\
91,105,119 \\
122,147,161 \\
175,188,246\end{array}$ & $\begin{array}{l}\text { 2H-Cyclohepta[b]furan-2-one,6-[1- } \\
\text { (acetyloxy)-3-oxobutyl]-3,3a,4,7,8,8a- } \\
\text { hexahydro-7-methyl-3-methylene- } \\
\left(\mathrm{C}_{17} \mathrm{H}_{22} \mathrm{O}_{5}\right)\end{array}$ & $\begin{array}{l}\text { Fused } \\
\text { heterocyclic } \\
\text { compound }\end{array}$ & 306 \\
\hline 15 & 32.1528 & 2454757.59 & $\begin{array}{l}69,81,105 \\
123,137,147 \\
177,199,207 \\
228,273,302\end{array}$ & $\begin{array}{l}\text { 4,8,13-Cyclotetradecatriene-1,3-diol,1,5,9- } \\
\text { trimethyl-12-(1-methylethyl)- } \\
\left(\mathrm{C}_{20} \mathrm{H}_{34} \mathrm{O}_{2}\right)\end{array}$ & $\begin{array}{l}\text { Substituted } \\
\text { cyclic } \\
\text { hydrocarbon }\end{array}$ & 306 \\
\hline 16 & 33.3718 & 735576.90 & $\begin{array}{l}69,97,109 \\
117,147,161 \\
175,191,207 \\
239,255,280 \\
340,407\end{array}$ & $\begin{array}{l}\text { 9,12,15-Octadecatrienoic acid-2,3- } \\
\text { bis(acetyloxy)propyl ester, }[\mathrm{Z}, \mathrm{Z}, \mathrm{Z}]- \\
\left(\mathrm{C}_{25} \mathrm{H}_{40} \mathrm{O}_{6}\right)\end{array}$ & $\begin{array}{l}\text { unsaturated } \\
\text { fatty acid }\end{array}$ & 436 \\
\hline
\end{tabular}

detected exclusively in CC-P extract (Table $1)$. On the other hand, Phenol,2,4-bis (1,1-dimethylethyl)-; 2-(4a,8-Dimethyl-6-oxo1,2,3,4-4a,5,6,8a-octahydro-naphthalen-2-yl) propionaldehyde; 4,8a-Dimethyl-6-(2-methyloxiran-2-yl)-4a, 5, 6, 7, 8, 8a-hexahydro-1Hnaphthalen-2-one; 2-[4-Methyl-6-(2,6,6trimethylcyclohex-1-enyl]hexa-1,3,5-trienyl] cyclohex-1-en-1-carboxaldehyde; Androstan3-one,17-hydroxy-1,17-dimethyl, $(1 \alpha, 5 \alpha, 17 \beta)$-; 2-Propenal,3-(2,6,6-trimethyl-1-cyclohexen1-yl)-; 2H-cyclohepta[b]furan-2-one,6[1-(acetyloxy)-3-oxobutyl]-3,3a, 4,7,8,8ahexahydro-7-methyl-3-methylene-; Dihydroxanthin; Stigmastane-3,6-dione, (5 $\alpha)$ and $\beta$-Sitosterol were exclusively detected in CC-C extract (Table 2). The abundance of a particular compound (\%) was calculated with respect to area (intensity height $x$ time) of corresponding peak relative to total area of entire chromatogram including both identified and unidentified peaks. Accordingly, the major constituents (relative abundance $>4 \%$ ) present in CC-P were cycloisolongifolence, 8,9-dehydro-9-formyl- (19.64\%), 4-oxo$\beta$-isodamascol $(16.05 \%), 6,10$-dimethyl3-(1-methylethyl)-6-cyclodecene-1,4dione $(13.80 \%), \quad 2 \mathrm{H}$-cyclohepta[b] furan-2-one, 6-[1-(acetyloxy)-3-oxobutyl]$3,3 \mathrm{a}, 4,7,8,8$ a-hexa hy d ro-7-methyl-3methylene- $(9.08 \%)$, tricycle $[5.1 .0 .0(2,4)]$ oct-5-ene-5-propanoic acid,3,3,8.8- tetramethyl- (6.39\%), 2 (3H)-benzofuranone, 6ethenylhexahydro-3,6-dimethy 1-7-(1methylethenyl)-, [3S- $(3 \alpha, 3 \mathrm{a} \alpha, 6 \alpha, 7 \beta, 7 \mathrm{a} \beta)$ $(4.18 \%)$ and 7 a-isopropenyl-4,5dimethyloctahydroindene-4-carboxylic acid $(4.01 \%)$. Similarly, the major constituents present in CC-C were cycloisolongifolence, 8,9-dehydro-9formyl-(15.69\%), 6,10-dimethyl-3-(1methylethyl)-6-cyclodecene-1,4-dione $(13.38 \%), \quad 2 \mathrm{H}-\mathrm{cyclohepta[b]furan-}$ 2-one,6-[1-(acetyloxy)-3-oxobutyl]$3,3 \mathrm{a}, 4,7,8,8$ a-hexahyd ro-7-methyl-3methylene- $(4.55 \%)$, tricycle $[5.1 .0 .0(2,4)]$ oct-5-ene-5-propanoic acid,3,3,8.8tetramethyl- $(7.50 \%), 2(3 \mathrm{H})$-benzofuranone, 6 ethenylhexahyd ro-3,6-dimethy 1-7-(1methylethenyl)-, [3S- $(3 \alpha, 3 \mathrm{a} \alpha, 6 \alpha, 7 \beta, 7 \mathrm{a} \beta)$ $(6.15 \%), \quad 7$ a-i sopropenyl-4,5dimethyloctahydroindene-4-carboxylic acid $(6.43 \%)$ and 2-(4a,8-Dimethyl-6-oxo1,2,3,4-4a,5,6,8a-octahydro-na phthalen-2yl) propionaldehyde $(6.57 \%)$. The list of the major constituents present in CC-P and CC-C extracts and their known bioactivities is given below.

i) Cycloisolongifolence,8,9-dehydro-9-formylwith $19.64 \%$ relative abundance (RA). It has antitumor, anti-plasmodial, and antiinflammatory activities (Lai et al. 2044; Lakshmi et al. 2011; Chadwick et al. 2013; Rita de Cássia et al. 2015) 
Table 2. GC-MS identification of phytochemicals present in chloroform extract (CC-C) of the rhizome of C. caesia

\begin{tabular}{|c|c|c|c|c|c|c|}
\hline $\begin{array}{l}\text { Sl. } \\
\text { No. }\end{array}$ & RT & $\begin{array}{l}\text { Area } \\
\text { [Intens sec] }\end{array}$ & $\begin{array}{l}\text { Mass } \\
\text { fragment } \\
\text { fingerprint } \\
(\mathrm{m} / \mathrm{z})\end{array}$ & Name of compound and molecular formula & $\begin{array}{l}\text { Class of } \\
\text { compound }\end{array}$ & $\begin{array}{l}\text { MW } \\
(\mathrm{Da})\end{array}$ \\
\hline 1 & 14.7525 & 11903149.65 & $\begin{array}{l}41,79,91 \\
108,133,148 \\
216\end{array}$ & $\begin{array}{l}\text { Benzofuran,6-ethenyl-4,5,6,7-tetrahydro-3,6- } \\
\text { dimethyl-5-isopropenyl-trans- } \\
\left(\mathrm{C}_{15} \mathrm{H}_{20} \mathrm{O}\right)\end{array}$ & Sesquiterpene & 216 \\
\hline 2 & 15.0057 & 3805507.39 & $\begin{array}{l}57,74,163 \\
191,206\end{array}$ & $\begin{array}{l}\text { Phenol,2,4-bis(1,1-dimethylethyl)- } \\
\mathrm{C}_{14} \mathrm{H}_{22} \mathrm{O}\end{array}$ & $\begin{array}{l}\text { Phenolic } \\
\text { compound }\end{array}$ & 206 \\
\hline 3 & 16.4312 & 70809565.21 & $\begin{array}{l}39,66,91,94 \\
122,123,162 \\
215,230\end{array}$ & $\begin{array}{l}\text { Cycloisolongifolene, 8,9-dehydro-9-formyl- } \\
\left(\mathrm{C}_{16} \mathrm{H}_{22} \mathrm{O}\right)\end{array}$ & Sesquiterpene & 230 \\
\hline 4 & 16.7176 & 29040931.29 & $\begin{array}{l}41,55,69,93 \\
107,121,136 \\
147,193,236\end{array}$ & $\begin{array}{l}\text { 7a-Isopropenyl-4,5- } \\
\text { dimethyloctahydroindene-4-carboxylic acid } \\
\left(\mathrm{C}_{15} \mathrm{H}_{24} \mathrm{O}_{2}\right)\end{array}$ & Sesquiterpene & 236 \\
\hline 5 & 17.7501 & 9953906.77 & $\begin{array}{l}41,53,67,79 \\
91,107,121 \\
135,136,147 \\
175,218\end{array}$ & $\begin{array}{l}\text { 3,7-Cyclodecadien-1-one,3,7-dimethyl-10-(1- } \\
\text { methylethylidene),(E,E)- } \\
\left(\mathrm{C}_{15} \mathrm{H}_{22} \mathrm{O}\right)\end{array}$ & Germacrone & 218 \\
\hline 6 & 18.0898 & 60415463.56 & $\begin{array}{l}41,55,69,82 \\
95,109,121 \\
139,165,167 \\
180,181\end{array}$ & $\begin{array}{l}\text { 6,10-Dimethyl-3-(1-methylethyl)-6- } \\
\text { cyclodecene-1,4-dione } \\
\left(\mathrm{C}_{15} \mathrm{H}_{24} \mathrm{O}_{2}\right)\end{array}$ & Sesquiterpene & 236 \\
\hline 7 & 18.2897 & 33944990.51 & $\begin{array}{l}41,55,67,79 \\
91,105,133 \\
165,189,191 \\
219,234\end{array}$ & $\begin{array}{l}\text { Tricyclo[5.1.0.0(2,4)]oct-5-ene-5-propanoic } \\
\text { acid,3,3,8.8-tetramethyl- } \\
\left(\mathrm{C}_{15} \mathrm{H}_{22} \mathrm{O}_{2}\right)\end{array}$ & Sesquiterpene & 234 \\
\hline 8 & 18.5495 & 10241547.94 & $\begin{array}{l}41,68,69,82 \\
95,109,121 \\
139,165,167 \\
180,181\end{array}$ & $\begin{array}{l}\text { Neocurdione } \\
\left(\mathrm{C}_{15} \mathrm{H}_{24} \mathrm{O}_{2}\right)\end{array}$ & Sesquiterpene & 236 \\
\hline 9 & 18.9958 & 29404606.41 & $\begin{array}{l}41,55,67,68 \\
95,96,109 \\
121,152,164 \\
178,191,219 \\
234\end{array}$ & $\begin{array}{l}\text { 2(3H)-Benzofuranone,6-ethenylhexahydro- } \\
\text { 3,6-dimethyl-7-(1-methylethenyl)-,[3S- } \\
(3 \alpha, 3 \mathrm{a} \alpha, 6 \alpha, 7 \beta, 7 \mathrm{a} \beta)] \\
\left(\mathrm{C}_{15} \mathrm{H}_{22} \mathrm{O}_{2}\right)\end{array}$ & Sesquiterpene & 234 \\
\hline 10 & 19.2622 & 5417179.54 & $\begin{array}{l}41,55,77,81 \\
94,107,122 \\
123,150,162 \\
215,230\end{array}$ & $\begin{array}{l}\text { Azuleno[4,5-b]furan-2(3H)- } \\
\text { one,3a,4,6a,7,8,9,9a,9b-octahydro-6-methyl- } \\
\text { 3,9-bis(methylene),[3aS-(3a } \alpha, 6 \mathrm{a} \alpha, 9 \mathrm{a} \alpha, 9 \mathrm{~b} \beta)]- \\
\left(\mathrm{C}_{15} \mathrm{H}_{18} \mathrm{O}_{2}\right)\end{array}$ & $\begin{array}{l}\text { Fused } \\
\text { aromatic } \\
\text { compound }\end{array}$ & 230 \\
\hline 11 & 19.7285 & 29680039.62 & $\begin{array}{l}43,53,68,79 \\
91,107,133 \\
161,176,177 \\
219,234\end{array}$ & $\begin{array}{l}\text { 2-(4a,8-Dimethyl-6-oxo- } 1,2,3,4- \\
\text { 4a,5,6,8a-octahydro-naphthalen-2-yl }) \\
\text { propionaldehyde } \\
\left(\mathrm{C}_{15} \mathrm{H}_{22} \mathrm{O}_{2}\right)\end{array}$ & Sesquiterpene & 234 \\
\hline 12 & 20.6478 & 7850033.86 & $\begin{array}{l}43,55,67,79 \\
91,105,121 \\
133,161,163 \\
173,197,212 \\
234\end{array}$ & $\begin{array}{l}\text { 6-(1-Hydroxymethylvinyl)-4,8a-dimethyl- } \\
\text { 3,5,6,7,8,8a-hexahydro-1H-naphthalene-2- } \\
\text { one } \\
\left(\mathrm{C}_{15} \mathrm{H}_{22} \mathrm{O}_{2}\right)\end{array}$ & Sesquiterpene & 234 \\
\hline
\end{tabular}




\begin{tabular}{|c|c|c|c|c|c|c|}
\hline 13 & 22.2265 & 20562711.13 & $\begin{array}{l}43,53,65,77 \\
91,105,119 \\
123,147,161 \\
175,188,203 \\
246\end{array}$ & $\begin{array}{l}\text { 2H-Cyclohepta(b)furan-2-one,6-[1- } \\
\text { (acetyloxy)-3-oxobutyl]-3,3a,4,7,8,8a- } \\
\text { hexahydro-7-methyl-3-methylene- } \\
\left(\mathrm{C}_{17} \mathrm{H}_{22} \mathrm{O}_{5}\right)\end{array}$ & Xanthinin & 306 \\
\hline 14 & 22.5195 & 12907768.89 & $\begin{array}{l}43,55,67,81 \\
107,122,149 \\
173,191,206 \\
219\end{array}$ & $\begin{array}{l}\text { 4,8a-Dimethyl-6-(2-methyl-oxiran-2-yl)- } \\
\text { 4a,5,6,7,8,8a-hexahydro-1H-naphthalen-2- } \\
\text { one } \\
\left(\mathrm{C}_{15} \mathrm{H}_{22} \mathrm{O}_{2}\right)\end{array}$ & Sesquiterpene & 234 \\
\hline 15 & 22.9259 & 6772960.15 & $\begin{array}{l}43,55,67,81 \\
107,123,136 \\
148,191,219\end{array}$ & $\begin{array}{l}\text { 2-[4-methyl-6-(2,6,6-trimethylcyclohex-1- } \\
\text { enyl]hexa-1,3,5-trienyl]cyclohex-1-en-1- } \\
\text { carboxaldehyde } \\
\left(\mathrm{C}_{23} \mathrm{H}_{32} \mathrm{O}\right)\end{array}$ & $\begin{array}{l}\text { Unsaturated } \\
\text { substituted } \\
\text { hydrocarbon }\end{array}$ & 324 \\
\hline 16 & 23.7519 & 3562571.26 & $\begin{array}{l}43,55,67,79 \\
91,105,131 \\
147,161,174 \\
175,189\end{array}$ & $\begin{array}{l}\text { Androstan-3-one,17-hydroxy-1,17- } \\
\text { dimethyl, }(1,5 \alpha, 17 \beta) \text { - } \\
\left(\mathrm{C}_{21} \mathrm{H}_{34} \mathrm{O}_{2}\right)\end{array}$ & Steroid & 318 \\
\hline 17 & 23.9650 & 5080956.50 & $\begin{array}{l}43,69,81,91 \\
105,145,175 \\
189,213,228 \\
246,247\end{array}$ & $\begin{array}{l}\text { 2-Propenal,3-(2,6,6-trimethyl-1-cyclohexen- } \\
\text { 1-yl)- } \\
\left(\mathrm{C}_{12} \mathrm{H}_{18} \mathrm{O}\right)\end{array}$ & $\begin{array}{l}\text { Ionone isomer } \\
\text { (terpene) }\end{array}$ & 178 \\
\hline 18 & 27.9951 & 7112246.57 & $\begin{array}{l}43,55,67,81 \\
95,110,124 \\
147,159,190 \\
205,230,248\end{array}$ & $\begin{array}{l}\text { Dihydroxanthin } \\
\left(\mathrm{C}_{17} \mathrm{H}_{24} \mathrm{O}_{5}\right)\end{array}$ & Terpene & 308 \\
\hline 19 & 37.6206 & 16989684.90 & $\begin{array}{l}43,69,81,95, \\
107,137,147, \\
175,189,207, \\
217,231,245, \\
246,247,287, \\
288,316,331, \\
359,385,399, \\
413,426\end{array}$ & $\begin{array}{l}\text { Stigmastane-3,6-dione, }(5 \alpha) \text { - } \\
\left(\mathrm{C}_{29} \mathrm{H}_{48} \mathrm{O}_{2}\right)\end{array}$ & Steroid & 428 \\
\hline 20 & 44.1753 & 2901572.24 & $\begin{array}{l}55,81,95, \\
107,145,161, \\
173,199,207, \\
213,239,255, \\
273,281,303 \\
329,355,381, \\
396,414\end{array}$ & $\begin{array}{l}\beta \text {-Sitosterol } \\
\left(\mathrm{C}_{29} \mathrm{H}_{50} \mathrm{O}\right)\end{array}$ & Sterol & 414 \\
\hline
\end{tabular}

ii) 4 -Oxo- $\beta$-isodamascol with $16.05 \%$ RA.

iii) 6,10-Dimethyl-3-(1-methylethyl)-6cyclodecene-1,4-dione with $13.80 \%$ RA

iv) $2 \mathrm{H}$-Cyclohepta[b]furan-2-one, 6-[1(a cetyloxy)-3-oxobutyl]-3,3a,4,7,8,8ahexahydro-7-methyl-3-methylene- with $9.08 \%$ RA.

v) Tricyclo $[5.1 .0 .0(2,4)]$ oct-5-ene-5-propanoic acid,3,3,8.8-tetramethyl with $6.39 \% \mathrm{RA}$. It has anticancer, Anti-plasmodial, and Antiinflammatory activities (Chadwick et al.
2013; Rita de Cássiaet al. 2015.

vi) $2(3 \mathrm{H})$-Benzofuranone,6-ethenylhexahydro3,6-dimethyl-7-(1-methylethenyl)-,,[3S$(3 \alpha, 3 \mathrm{a} \alpha, 6 \alpha, 7 \beta, 7 \mathrm{a} \beta$ with $4.18 \% \mathrm{RA}$.

vii) 7 a-Is opropeny $1-4,5$-dimethyloctahydroindene-4-carboxylic acid with $4.01 \%$ RA.

Componds iii,v,vi,vii also have anticancer, antiplasmodial, and antiinflammatory activities (Chadwick et al. 2013; Rita de Cássia et al. 2015). 
Major putative phytoconstituents identified by GC-MS in chloroform extract (CC-C) of the rhizome of $C$. caesia are

i.) Cycloisolongifolence,8,9-dehydro-9-formylwith $15.69 \%$ RA.

ii) 6,10-Dimethyl-3-(1-methylethyl)-6cyclodecene-1,4-dione with $13.38 \%$ RA

iii) 2H-Cyclohepta[b]furan-2-one, 6-[1(acetyloxy)-3-oxobutyl]-3,3a,4,7,8,8ahexahydro-7-methyl-3-methylene- with $4.55 \%$ RA

iv) Tricyclo [5.1.0.0(2,4)] oct-5-ene-5-propanoic acid,3,3,8.8-tetramethyl- with 7.50\% RA

v) 2(3H)-Benzofuranone, 6-ethenylhexahydro3,6-dimethyl-7-(1-methylethenyl)-,[3S$(3 \alpha, 3 \mathrm{a} \alpha, 6 \alpha, 7 \beta, 7 \mathrm{a} \beta)$ with $6.51 \% \mathrm{RA}$

v i ) 7 a - I s o p r o p e n y $1-4,5$ dimethyloctahydroindene-4-carboxylic acid with $6.43 \%$ RA

vii) 2-(4a,8-Dimethyl-6-oxo-1,2,3,4-4a,5,6,8aoctahydro-naphthalen-2-yl) propional dehyde with $6.57 \%$ RA.

All the compounds except compound (iii) have anti-cancer, anti-plasmodial, and antiinflammatory activities (Chadwick et al. 2013; Rita de Cássia et al. 2015).

Previous studies aimed at identifying phytochemicals from the essential oil of rhizome of $C$. caesia have indicated the predominance of sesquiterpenes, camphene, borylene, and $\delta$-camphor classes of compounds (Sastri 1962). However, the present study for the first time have compared GCMS profiling of $C$. caesia rhizome extracts of petroleum ether and chloroform and the major compounds present in these extracts were sesquiterpenes.

\section{Biocidal actions of extracts}

The results of antibacterial assay are shown in Table 3. Both the CC-C and CC-P showed inhibitory effect against all the bacterial test pathogens used in this study. The inhibition zone ranged from $10 \mathrm{~mm}$ to $18.25 \mathrm{~mm}$. In this study, Micrococcus luteus showed the highest sensitivity against CC-C and CC-P extracts with the nearly similar zone of inhibition of $\sim 18 \mathrm{~mm}$ whereas Bacillus subtilis showed the lowest sensitivity against both the extracts. The difference in the zone of inhibition against various pathogens might be due to the diffusing potency of the extract against the cell wall of a particular bacterium.

Further, the results of antifungal assay are shown in Table 4. Both the extracts (CC-C and CC-P) were found to show antifungal activity against Rhizoctonia solani with the nearly similar zone of inhibition of $\sim 10 \mathrm{~mm}$ but failed to show activity against Aspergillus niger. Above results thus suggested that the extracts exhibited broad spectrum activity against the pathogenic bacteria. The phytoconstituents such as flavonoids, and polyphenolic compounds are characteristically reported for contributing medicinal activities such as anti-oxidant, antiallergic, antimicrobial, anti-inflammatory and anti-cancer (Aiyelaagbe \& $\mathrm{O}$ samudiamen 2009). Similarly, anti-microbial activity of plant extract is known to be contributed by phytochemicals such as alkaloids, flavonoids, saponins, tannins, and steroids (Nethathe \& Ndip 2011). Since phytochemical screening of the CC-P and CC-C extracts confirmed the presence of all the above classes of chemicals, it justified their bioactivities.

\section{Evaluation of antioxidant activity}

\section{Neutralization of ABTS and DPPH radicals}

The outcomes of ABTS and DPPH assays suggested that the optical density (OD) of ABTS/DPPH solution decreased in the presence of the increasing concentrations of the extracts (CC-P/CC-C). This suggested the efficacy of extracts for neutralizing of ABTS and DPPH as antioxidants. The percentage (\%) neutralization or scavenging calculated from the decrease in absorbance of DPPH/ ABTS solution are shown in figures 2 A \& B respectively. From these graphs, the $\mathrm{IC}_{50}$ values for DPPH neutralization (concentration of extract that reduced the OD of DPPH solution to half of its initial value) estimated was $0.68 \pm 0.02$ 
Table 3. Antibacterial activity of the chloroform (CC-C) and petroleum ether (CC-P) extracts of the rhizomes of $C$. caesia. Values are mean of duplicate readings (mean $\pm \mathrm{SD}$ )

\begin{tabular}{|c|c|c|c|c|c|c|}
\hline \multirow{2}{*}{$\begin{array}{l}\text { Sl. } \\
\text { No }\end{array}$} & \multirow{2}{*}{$\begin{array}{l}\text { Crude } \\
\text { extract }\end{array}$} & \multirow{2}{*}{$\begin{array}{l}\text { Concentration } \\
\left(\mu \mathrm{g} \mathrm{mL} \mathrm{mL}^{-1}\right)\end{array}$} & \multicolumn{4}{|c|}{ Zone of inhibition (in $\mathrm{mm}$ ) } \\
\hline & & & $\begin{array}{l}\text { Bacillus } \\
\text { subtilis }\end{array}$ & $\begin{array}{l}\text { Micrococcus } \\
\text { luteus }\end{array}$ & Escherichia coli & $\begin{array}{l}\text { Mycobacterium } \\
\text { smegmatis }\end{array}$ \\
\hline 1 & CC-C & 25 & $10 \pm 0$ & $18 \pm 0$ & $16.75 \pm 0.75$ & $14.5 \pm 0.5$ \\
\hline 2 & CC-P & 25 & $12.5 \pm 0.5$ & $18.25 \pm 0.25$ & $13 \pm 0$ & $15 \pm 0$ \\
\hline 3 & Ampicillin & 25 & $25 \pm 0$ & $30.5 \pm 0.5$ & $28 \pm 1$ & $24 \pm 0$ \\
\hline
\end{tabular}

Table 4. Antifungal activity of the chloroform (CC-C) and petroleum ether (CC-P) extracts of the rhizomes of $C$. caesia. Values are mean of duplicate readings (mean \pm SD)

\begin{tabular}{lllll}
\hline Sl. & Crude extract & $\begin{array}{l}\text { Concentration } \\
\text { No }\end{array}$ & & \multicolumn{2}{c}{ Zone of inhibition (in mm) } \\
\cline { 3 - 5 } & & $\left(\mu \mathrm{gL}^{-1}\right)$ & Rhizoctonia solani & Aspergillus niger \\
\hline 1 & CC-C & 25 & $10.5 \pm 0.5$ & - \\
2 & CC-P & 25 & $10.5 \pm 0.5$ & - \\
3 & Fluconazole & 25 & $20.5 \pm 0.75$ & $17.5 \pm 0.5$ \\
\hline
\end{tabular}

$\mathrm{mg} \mathrm{mL} \mathrm{m}^{-1}$ and $0.13 \pm 0.01 \mathrm{mg} \mathrm{mL}^{-1}$ for CC-P and CC-C extracts, respectively (inset of Fig. 2A). Similarly, $\mathrm{IC}_{50}$ values of ABTS neutralization was $58.35 \pm 3.50$ and $36.60 \pm 2.00 \mu \mathrm{g} \mathrm{mL}^{-1}$ for CC-P and CC-C, respectively (inset of Fig. 2 B). The observed results revealed that the scavenging activity of CC-C extract for DPPH and ABTS radicals was $\sim 5$ times and $\sim 1.5$ times higher as compared to the CC-P extract.

\section{Inhibition of lipid peroxidation}

The $\gamma$-irradiation of the aqueous solution of liposomes facilitates oxidation of the lipid molecules through radiolytically generated hydroxyl radicals and forms malondialdehyde (MDA) as one of the final products of lipid per oxidation. The liposomal solution treated with a fixed concentration $\left(50 \mu \mathrm{g} \mathrm{mL}^{-1}\right)$ of CC-P and $\mathrm{CC}-\mathrm{C}$ extracts prior to $\gamma$-radiation showed
(A)

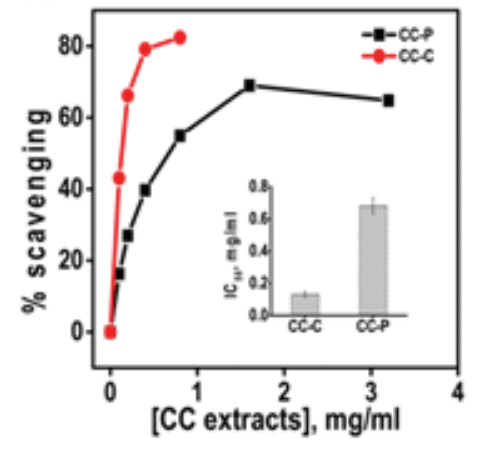

(B)

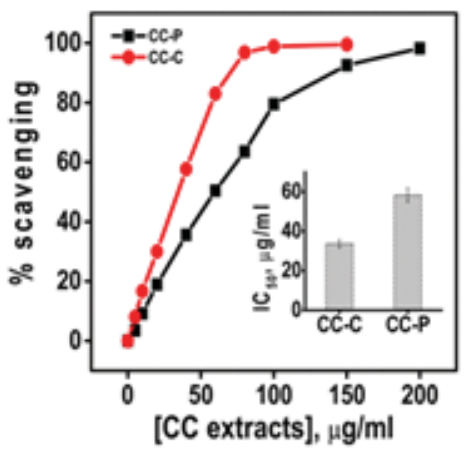

(C)

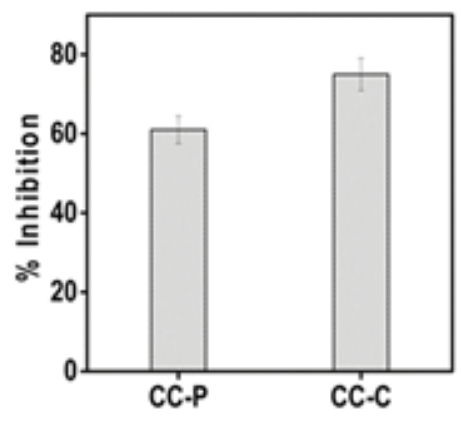

Fig. 2 (A) \& (B) Plots of percentage (\%) scavenging of DPPH and ABTS radical respectively vs concentration of extract. Inset of $(\mathrm{A})$ and $(\mathrm{B})$ show the $\mathrm{IC}_{50}$ values of extract obtained from DPPH and ABTS assays. (C) Plot of percentage (\%) inhibition of lipid peroxidation at fixed concentration $\left(50 \mu \mathrm{g} \mathrm{ml}^{-1}\right)$ of CC-P and CC-C extracts. Values are mean of duplicate readings (mean $\pm \mathrm{SD}$ ). 
decrease in the MDA content measured as TBARS as compared to untreated (liposomal solution without CC extracts) radiation control. The \% inhibition of lipid peroxidation calculated in terms of the decrease in the TBARS content was $61.0 \pm 3.5 \%$ and $75.0 \pm 4.1 \%$ respectively for CC-P and CC-C extracts (Fig. $2 \mathrm{C})$. This result suggested that CC-C extract was better than CC-P for inhibiting the lipid peroxidation which is in line with the results of DPPH and ABTS assays. The difference in the antioxidant activities of CC-P and CC-C extracts is attributed to the differential solubility of phytoconstituents between the polar and non-polar solvents.

\section{Conclusion}

The study documented 16 major volatile compounds in CC-P and 20 compounds in CC-C extracts. The extracts showed similar antibacterial and antifungal activities, but differed in anti-oxidant activities.

\section{Acknowledgements}

This work is a part of PhD thesis of Rameshor Singh Atom. The authors thank Sophisticated Analytical Instrument Facility of IIT Mumbai for GC-MS analysis and Rakhi Khunjamayum, Department of Biochemistry, Manipur University, Imphal for antimicrobial studies.

\section{References}

Aiyelaagbe O O 2009 Phytochemical screening for active compounds in Mangifera indica from Ibadan, Oyo State. Plant Sci. Res. 2: 11-13.

Arulmozhi D K, Sridhar N, Veeranjaneyulu A \& Arora S K 2006 Preliminary mechanistic studies on the smooth muscle relaxant effect of hydroalcoholic extract of Curcuma caesia. J. Herbal Pharmacotherapy 6(3/4): 117-124.

Astutik S, Jürgen P \& Jude N K 2019 Asian medicinal plants' production and utilization potentials: are view. Sustainability 11: 5483.

Atom R S, Warjeet S L, Rakhi K H, Shaukat A M S, Raghumani S N \& Kunwar A 2021 Evaluation of phytochemical, antioxidant and antimicrobial activities of Curcuma leucorrhiza (Zingiberaceae) Roxb. rhizome. IJPSR 12(9): 4764-4773.
Azwanida N N 2015 A Review on the extraction methods use in medicinal plants, principle, strength and limitation. Med. Aromat. Plants 4: 196.

Banerjee A \& Nigam S S 1976 Antifungal activity of the essential oil of Curcuma caesia Roxb. Indian J. Med. Res. 64: 1318-1321.

Bauer A W, Kirby W M M, Sherris J C \& Turck M 1966 Antibiotic susceptibility testing by a standardized single disk method. Am. J. Clin. Pathol.45: 493-496.

Bhupendra S, Rameshroo K \& Shashikant C 2016 Medicinal value of Curcuma cassia Roxb: an overview. UK J. Pharm. Biosci. 4 (6): 69-74.

Borah A, Paw M, Gogoi R, Loying R, Sarma N, Munda S, Pandey S K \& Lal M 2019 Chemical composition, antioxidant, anti-inflammatory, anti-microbial and invitro cytotoxic efficacy of essential oil of Curcuma caesia Roxb. leaves: an endangered medicinal plant of north east India. Ind. Crops. Prod. 129: 448-454.

Chadwick M, Trewin H, Gawthrop F \& Wagstaff C 2013 Sesquiterpenoids lactones: benefits to plants and people. Int. J. Mol. Sci. 14(6): 12780-12805.

Heisanam P, Mazumder P B \& Devi L P 2015 Antioxidant and antimutagenic activity of Curcuma caesia Roxb. rhizome extracts. Toxicol. Rep. 2: 423-428.

Donipati P \& Sreeramulu S H 2015 Preliminary phytochemical screening of Curcuma caesia. Int. J. Curr. Microbiol. App. Sci. 4 (11): 30-34.

Gupta V K, Kaushik A, Chauhan DS, Ahirwar R K, Sharma S \& Bisht D 2018 Anti- mycobacterial activity of some medicinal plants used traditionally by tribes from Madhya Pradesh, India for treating tuberculosis related symptoms. J. Ethnopharmacol. 227: 113-120.

I-Nan C, Chen-Chin C, Chang-Chai N, Chung-Yi W, Yuan-Tay S \& Tsu-Liang C 2008 Antioxidant and antimicrobial activity of Zingiberaceae plants in Taiwan. Plant Foods Hum. Nutr. 63: 15-20.

Jain A \& Parihar D K 2018 In vitro antioxidant and antidiabetic activity of regional chemotypes of three Curcuma species from Chhattisgarh. J. Herb. Sci. 7: 13-22. 
Jose S \& Thomas T D 2014 Comparative phytochemical and anti-bacterial studies of two indigenous medicinal plants Curcuma caesia Roxb. and Curcuma aeruginosa Roxb. Int. J. Green Pharm. 8: 65-71.

Kamazeri T S, Samah O A, Taher M, Susanti D \& Qaralleh H 2012 Antimicrobial activity andessential oils of Curcuma aeruginosa, Curcuma mangga, and Zingiber cassumunar from Malaysia. Asian. Pac. J. Trop. Med. 5: 202-209.

Karmakar I, Dolai N, Suresh Kumar R B, Kar B, Roy S N \& Haldar P K 2013 Antitumor activity and antioxidant property of Curcuma caesia against Ehrlich's ascites carcinoma bearing mice. Pharm Biol 2013; 51(6): 753-759.

Lai E Y, Chyau C C, Mau J L, Chen C C, Lai Y J, Shih C F \& Lin L L 2004 Antimicrobial activity and cytotoxicity of the essential oil of Curcuma zedoaria. Am. J. Chin. Med. 32: 281-90.

Lakshmi S, Padmaja G \& Remani P 2011 Antitumour effects of isocurcumenol isolated from Curcuma zedoaria rhizomes on human and murine cancer cells. Int. J. Med. Chem. 2011: 253962.

Mukunthan K S, Kumar A N V, Balaji S \& Trupti N P 2014 Analysis of essential oilconstituents in rhizome of Curcuma caesia Roxb. from south India. J. Essent. Oil-Bear. Plants 17: 647-651.

Nethathe B B \& N dip R N 2011 Bioactivity of Hydnoraafricana on selected bacterial pathogens: preliminary phytochemical screening. Afr. J. Microbiol. Res.518: 28202826.
Pancharoen O, Prawat U \& Tuntiwachwuttikul P 2000 Phytochemistry of the zingiberaceae. Stud. Nat. Prod. Chem. 23: 797-865.

Paw M, Gogoi R, Sarma N, Pandey S K, Borah A, Begun $\mathrm{T} \&$ Lal M 2020 Study of antioxidant, anti-inflammatory, genotoxicity, antimicrobial activities and analysis of different constituents found in rhizome essential oil of Curcuma caesia Roxb, collected from northeast India. Curr. Pharm. Biotechnol. 21 (5): 403-413.

Satyavam D A \& Warjeet S L 2012 Investigation of the structure-non linearity relationship of zederone from rhizome of Curcuma caesia Roxb. Indian J. Chem. 51: 1738-1742.

Rita de Cássia da Silveira e Sá, Andrade L N \& de Sousa D P 2015 Sesquiterpenes from essential oils and anti-inflammatory activity. Nat. Prod. Commun. 10 (10): 1767-1774.

Sastri B N 1962 The wealth of India - Raw materials. CSIR New Delhi.

Singh H, Krishna G, Baske P K 2010 Plants used in the treatment of joint diseases (rheumatism, arthritis, gout and lumbago) in Mayurbhanj district of Odisha, India. Report and Opinion 2(9): 22-26.

Sonjit D, Prodyut M, Zaman M K 2013 Curcuma caesia Roxb. and it's medicinal uses: a review. IJRPC 3(2): 370-375.

Voravuthikunchai S P 2007 Family Zingiberaceae compounds as functional antimicrobials, antioxidants and antiradicals. Food Global Science Books 1 (2): 227-240. 